\title{
E-LEARNING SYSTEM FOR THE COMPREHENSIVE LEARNING OF ELECTRONIC CIRCUITS
}

\author{
Atsushi Takemura \\ Tokyo University of Agriculture and Technology \\ 2-24-16, Naka-cho, Koganei-shi, Tokyo 184-8588, Japan
}

\begin{abstract}
This paper proposes a novel e-learning system for comprehensive learning covering theoretical analysis, design, and experiments involving the construction of practical electronic circuits. The proposed system possesses technical novelty that enables a student to efficiently construct circuits according to an automated instruction of circuit design. Moreover, this system allows the student to select an appropriate type of circuit construction strategy, such as with/without soldering, depending on his purpose and ability. The effectiveness of the proposed system was verified by performing a comparison experiment using 40 undergraduate students at the Tokyo University of Agriculture and Technology. The comparison tests were done by two groups of students comprising students that used or did not use the proposed system demonstrated the effectiveness of the system.
\end{abstract}

\section{KEYWORDS}

e-Learning, Experiment, Electronic Circuit Construction

\section{INTRODUCTION}

In technology education, acquiring knowledge and skills of electronic circuit construction and practical applications (e.g., signal processing, and machine control) are important. Therefore, it is necessary to teach and learn electronic circuit construction and how to perform experiments with the constructed circuits. However, it is difficult to efficiently teach and acquire comprehensive skills relating to the field of electronic circuitry such as theoretical analysis, design, and experiments involving circuit construction. Further, several university students in the world are presently forced to receive online lectures instead of normal classroom lectures because of the spread of COVID-19 in 2020. In the first semester, many Japanese universities were forced to embark on online lectures and did not open courses that require face-to-face instruction, such as experiments in a laboratory. To deal with these problems, the development of an e-learning system for virtual experiments to substitute laboratory works is needed. Recently, in the technology field, several remote laboratory systems have been developed for learning field-programmable gate array (FPGA) using augmented reality (AR) (Andujar, 2011), system control (Chevalier, 2017), and virtual measurements of electric circuits (Garcia-Zubia, 2017). However, these remote education systems are limited in scope and are only suitable for specific circuits within a subject area. To realize a remote learning system applicable to a wide variety of electronic circuits, a learning system for supporting laboratory works involving circuit construction based on image processing techniques was proposed (Takemura, 2019). However, this system is not applicable to e-learning and comprehensive electronic circuit learning. To overcome these shortcomings, this study proposes an e-learning system for the comprehensive learning of electronic circuits that covers theoretical analysis, design, and experiments involving the construction of practical electronic circuits. This system also has the technical novelty that enables individual students to select an appropriate level of circuit construction, such as with/without soldering on a circuit board, depending on the ability and purpose of the student.

The rest of this paper is organized as follows. Section 2 presents the technical features of the proposed system. Section 3 presents the experimental methodology to evaluate the proposed system. Section 4 presents the quantitative evaluation of the proposed system based on the experiments performed by actual university students. Finally, Section 5 presents the conclusions of the study. 


\section{TECHNICAL FEATURES OF THE SYSTEM}

To realize an e-learning system for the comprehensive learning of electronic circuits, this study develops two functions described in 2.1 and 2.2.

\subsection{Function for Learning Design and the Workings of Circuits}

Figure 1(a) shows a schematic of the function for learning circuit design and the workings of electronic circuits (F-1). The function F-1 helps the student in learning theoretical analysis, design, and understanding of the workings of electronic circuits through circuit simulation. F-1 translates a designed circuit into the general circuit description language, simulation program integrated circuit emphasis (SPICE) (Rabaey). F-1 possesses technical novelty needed in performing an automated translation technique involving cooperation with the function for virtual experiments (described in 2.2) and the evaluation of a designed circuit based on the SPICE information in the system's database. F-1 allows a student to learn design and the working of circuits in the following manner $A$ ) $-C$ ).

\section{A) Web-based Instruction}

The proposed system provides individual students with manuals and the datasheets necessary for understanding the theoretical analysis of circuit design available through common databases in the system.

\section{B) Circuit Design and Evaluation}

Students can calculate the parameters of the circuit components (e.g., connected devices and equipment) based on the web-based instruction of theoretical analysis of the system. Following this, the student can use his PC to draw the circuit diagram using a general circuit simulator (e.g., LTspice) using the SPICE-based processing. The SPICE-based simulator translates the circuit designed by a student into SPICE. This function can automatically evaluate the designed circuit by analyzing the differences in the SPICE information between a designed circuit and the model circuit prepared in the database of the proposed system. Based on this evaluation, the system automatically indicates errors in the designed circuits and instructs the students to correct the errors.

\section{C) SPICE-Simulation and Automated Evaluation}

Based on SPICE information, students can simulate the working of the designed circuit. The system requires the students to check whether the simulated working of his designed circuit corresponds to the specification and working of the model circuit in the database.

\subsection{Function for Virtual Experiments Involving Circuit Construction}

Figure 1(b) schematizes the function for learning virtual experiments involving circuit construction (F-2). The function F-2 helps a student in web-based learning of circuit construction and measurements of the constructed circuits. To virtually construct a circuit, F-2 allows individual students to use general graphic editors in their PCs to virtually construct circuits and performs automated translation of the virtual circuits into SPICE. This study improves on the previous SPICE-translation technique (Takemura, 2019) by making it possible to perform automated evaluation of the parameters of circuit components. F-2 automatically evaluates the virtually constructed circuit by comparing it with the SPICE information of the model circuit in the database of the system. Further, F-2 possesses the technical novelty and wide usefulness that enables individual students to select an appropriate type of circuit board (a breadboard or universal circuit board) depending on their purposes and abilities. Circuit construction using a breadboard does not require soldering of the circuit components (e.g., devices and cables), and the components' terminals are only plugged into the holes on the upper surface of the breadboard. Therefore, breadboard-based construction is suitable for beginners' education because of the easiness of construction and reusability of circuit components. However, the solder-less circuit on a breadboard is inferior in terms of stability and practical use. Thus, the breadboard is only applicable to trial construction or construction of small-scale circuits for elementary education. On the other hand, circuit construction using a 
universal circuit board necessitates soldering on the lower surface of the circuit board; thus, circuit construction on a universal circuit board is suitable for students whose experiences and abilities of circuit construction are at intermediate level and above. Further, circuit construction on a universal circuit board with soldering is applicable to practical circuits and large-scale circuits. The approach for learning virtual circuit construction using F-2 is presented in the subsections $A$ ) and $B$ ) below.

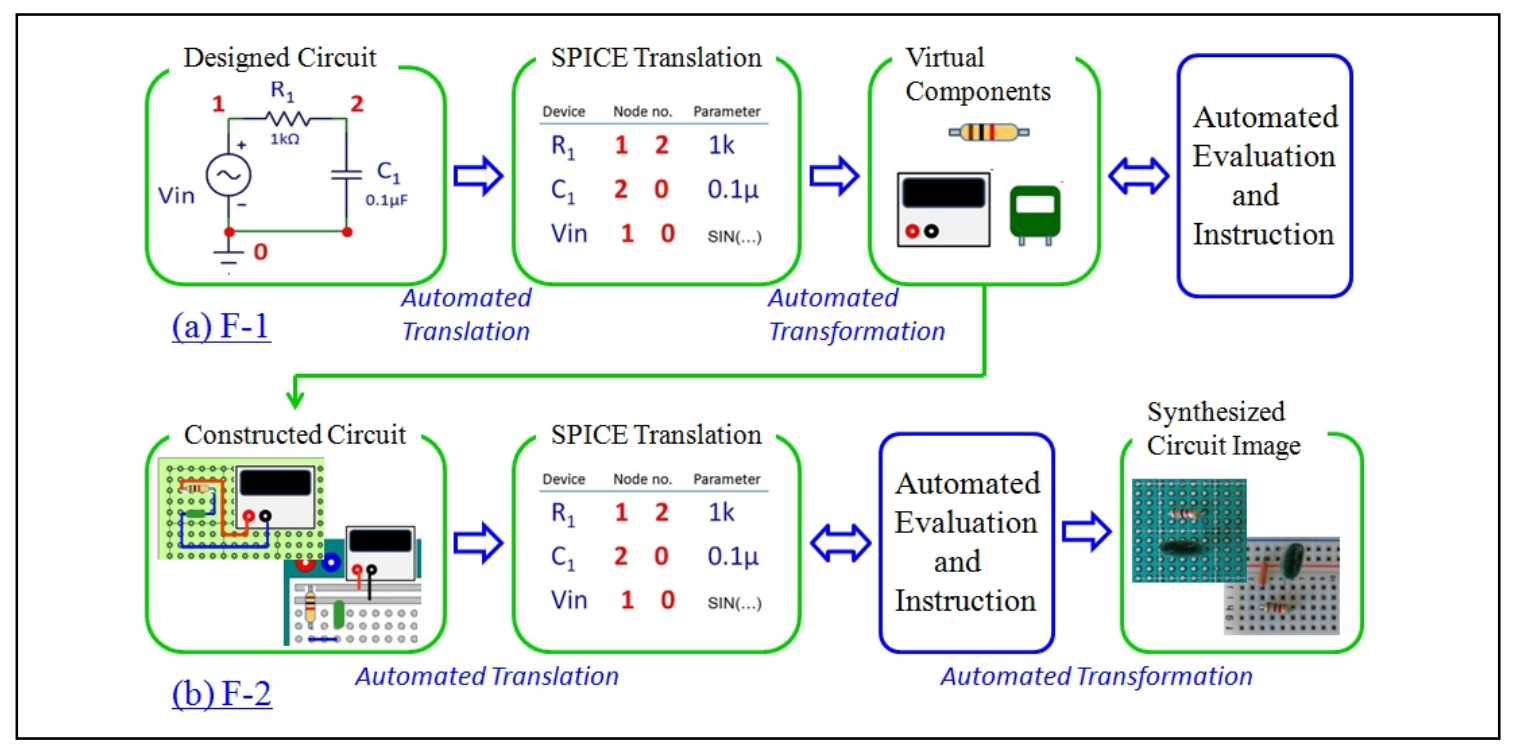

Figure 1. Schematic of the two proposed functions for experiments involving circuit construction; (a) function for learning design and workings of circuits (F-1) and (b) function for virtual experiments involving circuit construction $(\mathrm{F}-2)$

\section{A) Virtual Circuit Construction and Evaluation}

The manner of experiments involving the virtual circuit construction using each circuit board is presented hereunder.

\section{- Virtual Circuit Construction on a Breadboard}

The function F-2 allows individual students to construct virtual circuits using general graphical editors in their PCs. F-2 automatically provides the student with the pictures of necessary circuit components corresponding to the SPICE information obtained from the circuit designed using F-1 (see Figure 1). To construct a virtual circuit, the student places the virtual components on a picture of the selected circuit board and draws lines to indicate the circuit connections. Following this virtual construction, F-2 automatically translates the composition of the virtual circuit into SPICE. This SPICE information allows the student to simulate the workings of the virtual circuit and learn measurements of the circuit. The presence and location of incorrect components in the virtual circuit are determined by analyzing the difference in SPICE information between the constructed circuit and the correct circuit in the database of the system. If faulty parts or incorrect behavior of the virtual circuit is observed, the system provides the student with instructions or warnings on the issue.

\section{- Virtual Circuit Construction on a Universal Circuit Board with Soldering}

F-2 allows a student to use a general graphic editor in his PC and enables him to design the layout of a circuit by placing the virtual circuit components obtained from F-1 on the image of the upper surface of a universal circuit board. The student draws colored lines to indicate the wiring of each device on the upper surface of the circuit board. Based on the circuit recognition of an image of the upper surface of the virtual circuit, the proposed system can automatically provide instructions for wiring and soldering on the lower surface of the circuit board. F-2 also simulates the circuit operation based on automated translation of the constructed circuit into SPICE and individual students can virtually learn measurements of their circuit. The presence and location of incorrect components in the virtual circuit are determined by analyzing the difference 
in the SPICE information between the constructed circuit and the correct circuit in the system's database. If faulty parts or incorrect behavior of the virtual circuit is observed, the system provides the student with instructions or warnings on the issue.

\section{B) Image Synthesis for Learning a Physical Circuit}

To learn physical circuit-construction using the proposed e-learning system, F-2 provides the students with a synthesized image of the physical circuit by using the images of physical circuit-components corresponding to the virtual components during the process $A$ ). This synthesized image gives the student knowledge of the actual circuit construction using physical circuit components.

\section{METHODOLOGY FOR EVALUATING THE PROPOSED SYSTEM}

The effectiveness of the proposed e-learning system was evaluated via a comparison experiment by two groups of students aged 19-21 at Tokyo University of Agriculture and Technology. Group-1 comprised 20 students instructed to construct a DC voltage control circuit shown in Figure 2(a), by using conventional methods. Group- 2 consisted of 20 students who performed the same circuit construction using the proposed e-learning system. Groups-1 and -2 were evaluated in 2019 and 2020, respectively. Before starting the experiments, questionnaires were provided to ascertain the degrees of individual students' experience in circuit construction with/without soldering. Based on this, the students were subdivided into three categories as listed below:

A: Experienced in circuit construction with soldering in addition to practice exercises at school

B: Experienced in breadboard-based circuit construction in addition to practice exercises at school but no experience in circuit construction with soldering except for practice exercises at school

C: No experience in circuit construction except for practice exercises at school

The number of students belonging to each experience category is shown in Figure 2(b) and indicates that more than half of the students belonged to Category $\mathrm{C}$ and the majority of the students have no experience in circuit construction on a universal circuit board with soldering except for practice exercises at school. All students had learned the construction of elementary circuits with soldering as a part of compulsory education in junior high schools in Japan and thus, there were no students with no experience in circuit construction.

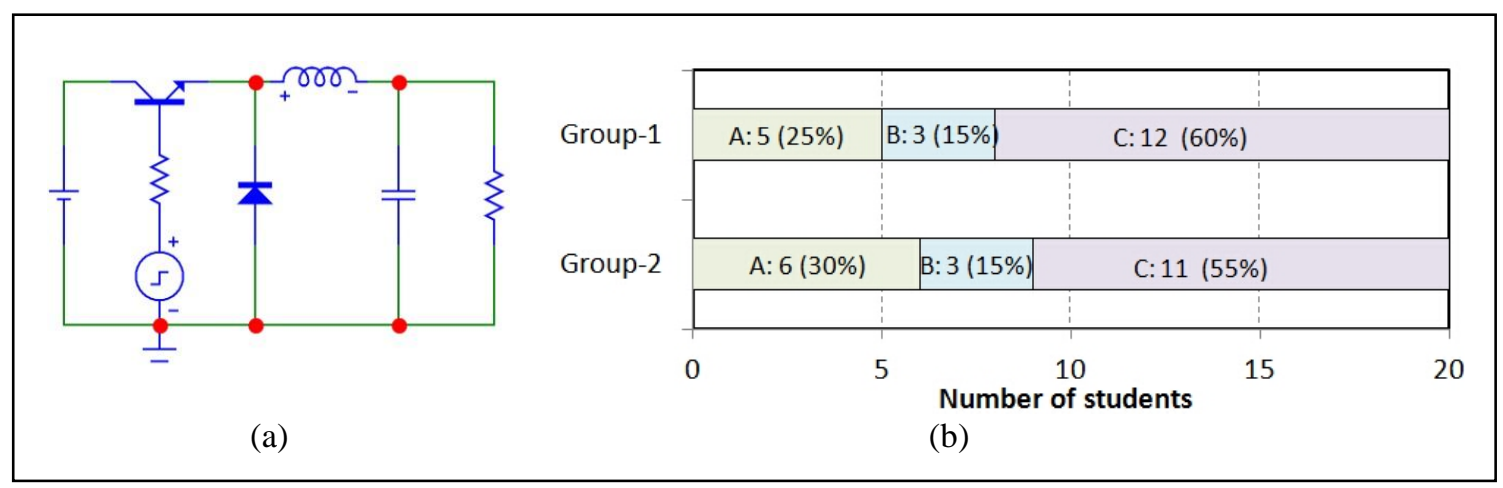

Figure 2. Circuit and grouping of students for evaluation of the proposed system; (a) circuit diagram to be constructed and (b) distribution of experience categories in circuit construction for each group

The students in Group-1 were tasked to construct circuits based on conventional methods listed below within a time limit of $4 \mathrm{~h}$ :

(G1-1) Lecture on the theory and analysis of a DC voltage controller circuit by an instructor $(0.5 \mathrm{~h})$

(G1-2) Theoretical analysis and circuit design

(G1-3) The instructor inspects the result of (G1-2). If an incorrect part is discovered, the concerned student corrects it under the instructor's guidance (1 h for (G1-2) and (G1-3))

(G1-4) Physical circuit construction using the necessary circuit components and a breadboard provided by the instructor 
(G1-5) The instructor inspects the constructed circuit. If an incorrect part is discovered, this student corrects it under the instructor's guidance ( $1.5 \mathrm{~h}$ for (G1-4) and (G1-5))

(G1-6) Measurement of the characteristics of the constructed circuit using experimental equipment $(1 \mathrm{~h})$

The individual students in Group-2 were tasked to construct circuits using the proposed new system within $4 \mathrm{~h}$ according to the following processes.

(G2-1) Online lecture on the theory and analysis of a DC voltage controller circuit by an instructor $(0.5 \mathrm{~h})$

(G2-2) Theoretical analysis and circuit design using F-1

(G2-3) The system automatically inspects the result of (G1-2). If an incorrect part is discovered, this student corrects it based on the instruction from F-1 (1 h for (G2-2) and (G2-3)).

(G2-4) Virtual circuit construction using F-2-each student can choose an appropriate circuit board (a breadboard or universal circuit board) depending on his purpose and ability.

(G2-5) The system automatically inspects the virtually constructed circuit. If an incorrect part is discovered, the concerned student corrects it based on the instruction from F-2 (1.5 h for (G2-4) and (G2-5)).

(G2-6) Measurement of the characteristics of the virtually constructed circuit using F-2 (1 h)

\section{RESULTS AND DISCUSSION}

In this section, the effectiveness of the proposed e-learning system is discussed based on the results of the comparison test between Group-1 and Group-2 as described in Section 3. Figure 3(a) shows examples of the web-based experiments on the virtual circuit construction using the proposed e-learning system obtained by a student in Group-2. This student was able to complete the two types of virtual circuit constructions using a breadboard and a universal circuit board with soldering and virtual measurements using these two circuits within the time limit.

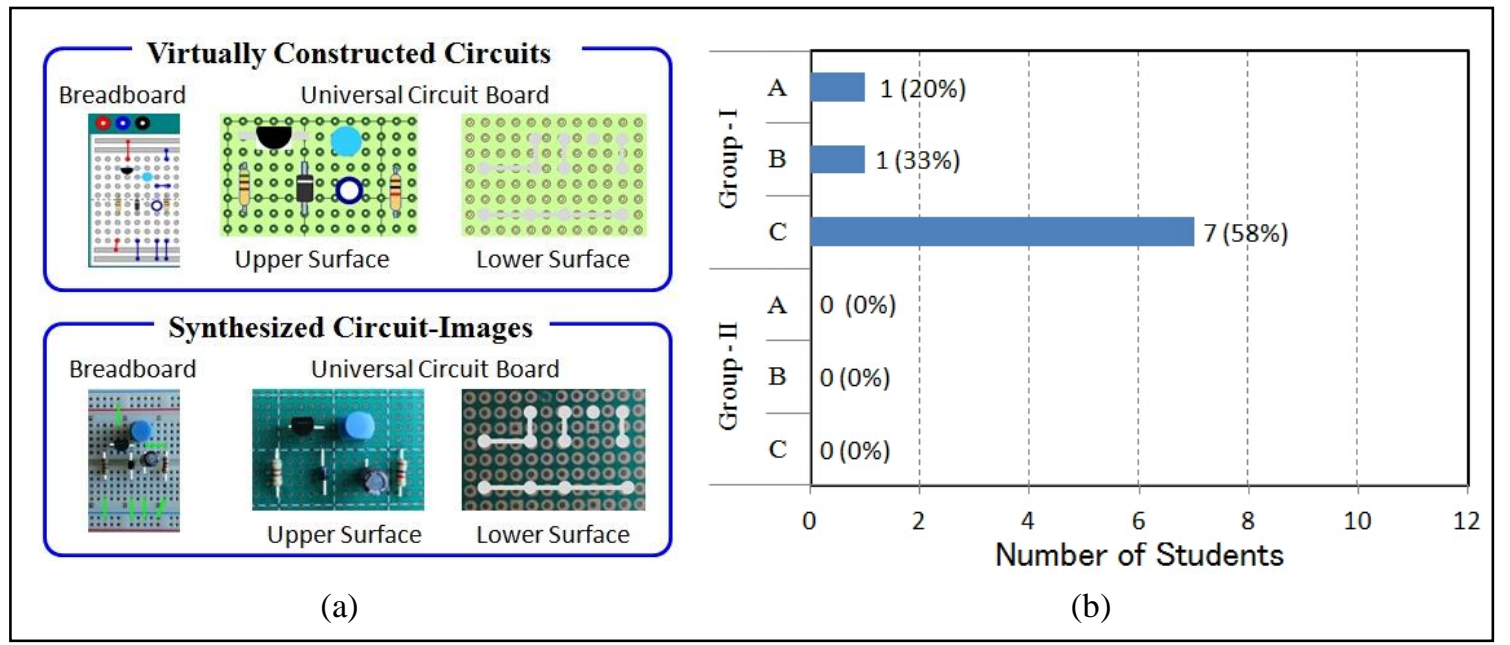

Figure 3. Results of the comparison test between Group-1 and Group-2; (a) virtual circuits constructed by a student in Group- 2 using the proposed system and (b) the number of students whose circuits contained errors.

Figure 3(b) indicates the occurrence of errors in the constructed circuits that were discovered at the processes (G1-5) and (G2-5). As shown in this figure, nine (45\%) students in Group-1 had errors in circuit construction that were observed by the instructor. Conversely, no errors in the circuits constructed by the students in Group-2 were noted. These results indicate the effectiveness of the proposed system for circuit construction. It is worthy of note that all students in Group-2 were able to complete two types of circuit construction using a breadboard and universal circuit board with soldering within the same time limit as Group-1.

Through the evaluation process, positive responses were obtained from the instructors, which provide further validation of the effectiveness of the proposed system. These comments show that: 
- This new system enables e-learning for teaching and learning both theory and experiments.

- This system has wide usability because individual students can learn circuit construction using an appropriate type of circuit boards depending on their abilities and purposes.

- The web-based instructions decrease an instructor's load for instructing experiments involving circuit construction.

From the students in Group-2, positive responses indicating the usefulness of the proposed system were obtained, as follows:

- The system was helpful in checking whether the workings of designed and constructed circuits are correct or not and in removing errors in the circuit.

- Circuit design using F-1 has an important role for improving the efficiency of circuit construction.

- F-2 is useful because an appropriate circuit board is selectable depending on user's purpose and ability. This function helps beginners to challenge an advanced level of circuit construction (e.g., soldering and large-scale circuit).

- The synthesized imaging of this e-learning system provides useful knowledge of the real shape of a physical circuit.

To improve the applicability and usefulness of the system, the following studies should be implemented:

- Improvement of the graphic quality using 3D-CG

- Expansion of the applicability to various use cases by combining virtual reality (VR) and AR.

\section{CONCLUSION}

A novel e-learning system for comprehensive learning of electronic circuits covering theoretical analysis, design, and experiments involving circuit construction is proposed. The proposed system enables students to select an appropriate mode of circuit construction depending on their purposes and abilities. A comparison study between two groups of university students demonstrated the effectiveness of the proposed system for accurate circuit construction with and without soldering on a circuit board. This e-learning system is expected to be applicable to various use cases by improving graphics qualities such as 3D graphics, VR, and AR.

\section{ACKNOWLEDGMENT}

This study was partly supported by a Grant-in-Aid for Scientific Research (KAKENHI) 19K03079 from the Japan Society for the Promotion of Science.

\section{REFERENCES}

Andujar, J. M, et al., 2011, Augmented reality for the improvement of remote laboratories: an augmented remote laboratory, IEEE Trans. Education, vol.54, no. 3, pp. 492-500.

Chevalier, A., et al., 2017, A three-year feedback study of a remote laboratory used in control engineering studies, IEEE Trans. Education, vol. 60, no. 2, pp. 127-133.

Garcia-Zubia, J., et al., 2017, Empirical analysis of the use of the VISIR remote lab in teaching analog electronics, In IEEE Trans. Education, vol. 60, no. 2, pp. 149-156.

Rabaey, J. M., The Spice Page, <URL: http://bwrc.eecs.berkeley.edu/Classes/IcBook/SPICE/> (accessed Aug. 31, 2020).

Takemura, A., 2019, Education system for electronic circuit construction involving soldering on a circuit board, Proc. IADIS Conference CELDA 2019, pp. 447-452, Cagliari, Italy. 